20

\title{
Рассеяние лазерного пучка на ансамбле асимметричных эритроцитов
}

\author{
(C) С.Ю. Никитин ${ }^{1}$, В.Д. Устинов ${ }^{2}$, Е.Г. Цыбров ${ }^{3, \uparrow}$, М.С. Лебедева ${ }^{1}$ \\ 1 Московский государственный университет им. М.В. Ломоносова, фризический факультет, \\ 119991 Москва, Россия \\ ${ }^{2}$ Московский государственный университет им. М.В. Ломоносова, центр фундаментальной и прикладной математики, \\ 119991 Москва, Россия \\ ${ }^{3}$ Московский государственный университет им. М.В. Ломоносова, факультет вычислительной математики и кибернетики, \\ 119991 Москва, Россия \\ ฯ e-mail: tsybrovevgeniy@yandex.ru
}

Поступила в редакцию 27.10.2020 г.

В окончательной редакции 27.12.2020 г.

Принята к публикации 22.02.2021 г.

\begin{abstract}
Построена аналитическая модель рассеяния лазерного пучка на неоднородном ансамбле эритроцитов. Модель учитывает различие эритроцитов по размерам, формам и ориентациям в пространстве. Установлена связь экспериментально измеряемого параметра - видности дифракционной картины - с характеристикой, имеющей смысл меры неоднородности образца крови по размерам и формам эритроцитов. Показано свойство монотонности этой зависимости, что позволяет оценивать разброс эритроцитов по размерам и формам на основе измерения видности дифракционной картины.
\end{abstract}

Ключевые слова: дифракционная картина, эритроцит, видность, распределение по размерам.

DOI: $10.21883 /$ OS.2021.07.51089.269-20

\section{Введение}

Лазерную дифрактометрию мазка крови можно рассматривать как метод измерения распределения эритроцитов по размерам [1]. Для проведения таких измерений необходимо просветить мазок крови лазерным пучком, сфотографировать возникающую при этом дифракционную картину и обработать ее на компьютере с помощью специального алгоритма. Достоинством этого метода являются его относительная простота, а также возможность быстро обрабатывать большие ансамбли клеток крови без использования микроскопа. Алгоритм обработки дифракционной картины строится на основе решения физической задачи о рассеянии лазерного пучка на неоднородном ансамбле частиц. Эта задача включает в себя определенную модель ансамбля эритроцитов. В работе [2] мы моделировали эритроциты на мазке крови круглыми дисками, отличающимися друг от друга по радиусу. Более точная модель должна учитывать особенности формы эритроцитов. В настоящей работе мы моделируем ансамбль эритроцитов на мазке крови набором плоских эллиптических дисков с разбросом по размерам, формам и ориентациям. Наша основная цель - связать статистические характеристики такого ансамбля с параметрами наблюдаемой дифракционной картины.

Касаясь медицинского аспекта проблемы, отметим, что важным параметром крови является разброс эритроцитов по размерам (RDW - Red Blood Cell Distribution Width). Известно, что повышенное значение этого параметра (более 15\%) является неспецифическим факто- ром, указывающим на общее неблагополучное состояние организма человека [3]. Повышенное значение RDW рассматривают как неблагоприятный фактор при заболеваниях сердца и периферических сосудов, болезнях почек и печени [4], а также при остром тяжелом воспалении легких, вызванном короновирусной инфекцией (COVID-19) [5].

\section{Лазерная дифрактометрия как метод измерения размеров частиц}

Измерения размеров малых частиц на основе лазерной дифракции ведут свою историю с 80-х годов прошлого века [6]. В настоящее время эта методика применяется для контроля качества порошков, состоящих из малых частиц. Это лекарственные и химические препараты, биологические клетки, взвесь ила речного дна, микросферы краски и многие другие [7]. Однако в большинстве работ по данной методике каждая частица моделируется с помощью сферы или тонкого круглого диска, поэтому реальные несферические частицы моделируются с ошибкой. Это не является критическим препятствием для применения методики, так как она все равно позволяет получать повторяемые результаты на схожих и различные результаты на существенно отличающихся образцах малых частиц. Тем не менее в ряде областей, например в медицинской диагностике крови, крайне важно представлять результат измерения в адекватном виде [8]. Для этого необходимы более совершенные модели рассеяния лазерного излучения 
на неоднородных ансамблях частиц [9]. Одна из таких моделей представлена в настоящей работе.

В медицине наиболее распространенными методами для измерения размеров эритроцитов считаются счетчик Коултера и стандартная оптическая микроскопия [8]. Оба этих метода имеют свои достоинства и недостатки, но здесь лишь отметим, что для увеличения количества эритроцитов, размеры которых требуется определить, необходимо прямо пропорционально увеличить и длительность измерения. В лазерной дифрактометрии, чтобы увеличить количество эритроцитов, участвующих в измерении, необходимо лишь расширить лазерный пучок. В частности, количество освещаемых эритроцитов может быть от десятков тысяч до миллиона в одном акте измерения, длящемся порядка секунды, включая компьютерную обработку данных. Отметим, что помимо проведения самого измерения необходимо заранее приготовить образец крови на мазке, что может занимать значительно больше времени. Тем не менее возможность быстрой обработки большого ансамбля эритроцитов является принципиальным преимуществом метода лазерной дифракции по сравнению с конкурирующими аналогами. Кроме того, данные лазерной дифрактометрии могут дополнять и уточнять данные измерений счетчика Коултера и оптической микроскопии.

Измерения лазерной дифракции начали применять для анализа эритроцитов относительно недавно [10-13]. Известен целый ряд работ, посвященных решению только прямой задачи рассеяния плоской монохроматической волны на одиночной частице несферической формы, имитирующей эритроцит [14-17]. В прямой задаче известны все параметры лазерного пучка и характеристики частицы, и требуется рассчитать дифракционную картину.

Решение прямой задачи обычно используют как готовый компонент для решения обратной задачи, в которой дана дифракционная картина и необходимо найти функцию распределения частиц по размерам. В обратной задаче предполагается, что исследуемые частицы освещены лазером с известной длиной волны, и сфотографирована дифракционная картина, из которой можно извлечь угловое распределение интенсивности света, рассеянного на этих частицах. Дифракция измеряется в дальней зоне в малых углах (примерно до $15-25^{\circ}$ отклонения от направления лазерного луча в зависимости от постановки задачи). Решению этой задачи в общем виде посвящено много работ (см., например, обзор [7]). Существуют приборы, известные как лазерные дифрактометры, лазерные гранулометры, или, в английской литературе, laser particle sizers, доступные коммерчески [18].

Хотя производители не раскрывают некоторые особенности своих приборов, в целом все они следуют одному и тому же принципу. Обратная задача сводится к решению интегрального уравнения Фредгольма первого рода следующего вида [7]:

$$
\int_{r_{1}}^{r_{2}} \tilde{I}(r, \theta) w(r) d r=I(\theta),
$$

где $w(r)$ - искомое распределение частиц по размерам. Функция $\tilde{I}(r, \theta)$ описывает угловое распределение интенсивности света, рассеянного на одной круглой или сферической частице с радиусом $r$. Функция $I(\theta)$ есть угловое распределение интенсивности света, рассеянного на всем ансамбле частиц. Эта функция измеряется экспериментально. Данная задача считается некорректно поставленной в том смысле, что ее решение неустойчиво и сильно зависит от шумов [19]. Для ее решения применяют различные методы регуляризации [20,21].

Если в указанном интегральном уравнении заменить интеграл на интегральную сумму с конечным числом слагаемых, а также ввести конечный набор углов наблюдения $\theta_{j}$, получим систему линейных алгебраических уравнений:

$$
\sum_{i=1}^{N} \tilde{I}\left(r_{i}, \theta_{j}\right) p_{i}=I_{j}, \quad 1 \leq j \leq M,
$$

где $p_{i}=p\left(r_{i}\right)$ обозначает вероятность того, что радиус эритроцита равен $r_{i}, I_{j}=I\left(\theta_{j}\right)$ обозначает интенсивность света, рассеянного под углом, равным $\theta_{j}$. Решая эту систему уравнений, в принципе можно найти всю функцию распределения эритроцитов по размерам, но для этого понадобится измерение распределения интенсивности света в широкой области дифракционной картины. Если же ограничиться измерением интенсивности лишь в двух углах (в темном кольце и в светлом кольце дифракционной картины), то можно найти первые два момента функции распределения эритроцитов по размерам [2,22].

Типичный вид мазка крови под микроскопом показан на рис. $1, a$. На рис. $1, b$ изображена типичная дифракционная картина, соответствующая рассеянию лазерного пучка с длиной волны $650 \mathrm{~nm}$ на влажном мазке эритроцитов. В центре дифракционной картины всегда наблюдается характерное круглое светлое пятно, по мере удаления от центра видно темное кольцо, еще дальше светлое кольцо, и так далее темные и светлые кольца чередуются друг с другом. В целом структура напоминает известную картину Эйри (картину рассеяния плоской волны на круглом отверстии) [23]. Видность $v$ такой картины определяется формулой [2]:

$$
v=\frac{I_{\max }-I_{\min }}{I_{\max }+I_{\min }},
$$

где $I_{\min }-$ значение интенсивности в первом минимуме дифракционной картины, а $I_{\max }$ - значение интенсивности в последующем максимуме дифракционной картине. Видность дифракционной картины можно использовать для оценки разброса частиц по размерам [2]. 


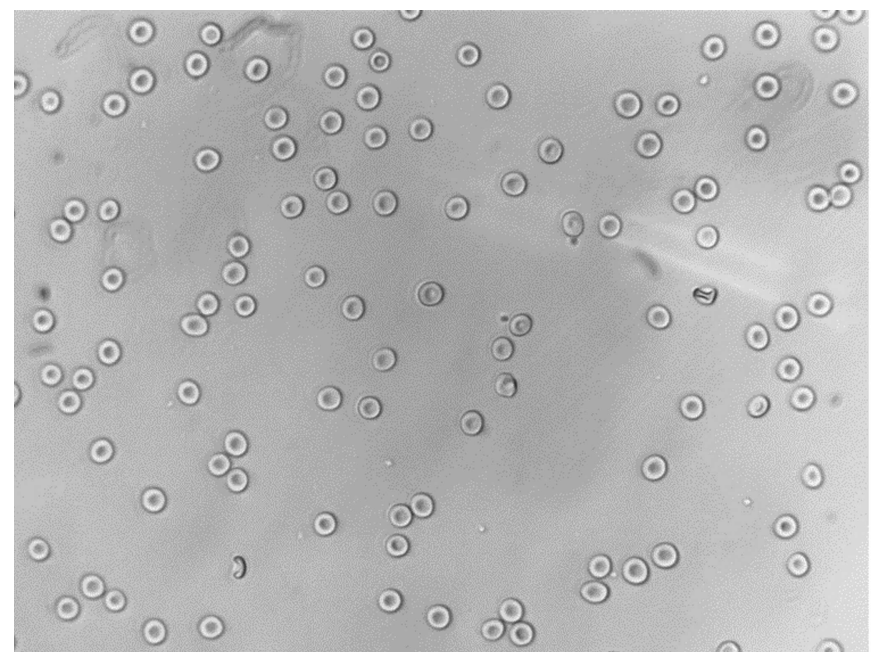

$b$

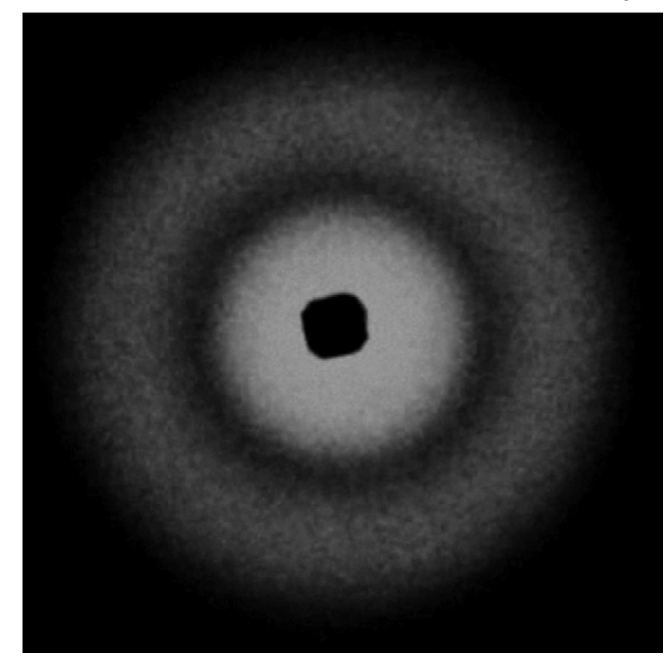

Рис. 1. Вид мазка крови под микроскопом $(a)$ и картина рассеяния лазерного пучка на мазке крови $(b)$.

В работах [1,2,22] мы моделировали эритроциты круглыми дисками. В настоящей работе мы обобщаем теорию, рассматривая частицы более сложных форм. Сначала мы представим теоретическую модель, описывающую рассеяние лазерного пучка на частицах эллиптической формы со случайными ориентациями в плоскости предметного стекла. Затем введем меру асимметрии частиц произвольной формы. Наконец, опишем численные расчеты, направленные на проверку теории. С математической точки зрения наш подход близок к подходу, развитому в работах [24-26]. Предлагаемый нами анализ отличается тем, что он чувствителен к формам эритроцитов на мазке крови. Такие анализы позволят оценивать долю серповидных клеток, эхиноцитов, пойкилоцитов и клеток других неправильных форм, которые по разным причинам могут присутствовать в исследуемом образце крови.

\section{Рассеяние лазерного пучка на ансамбле эллиптических дисков}

На рис. 1 показаны вид мазка крови под микроскопом и картина рассеяния лазерного пучка на нем (дифракционная картина). Видно, что эритроциты отличаются друг от друга по размерам и формам. Они не являются идеально круглыми дисками. Ориентацию эритроцита на мазке можно задать прямой линией, проведенной через две наиболее удаленные друг от друга точки эритроцита. Для эритроцита эллиптической формы эта линия направлена вдоль большой оси эллипса. Мы полагаем, что эта ориентация случайна. Типичная дифракционная картина обладает круговой симметрией и представляет собой систему концентрических темных и светлых колец. Известно, что радиус первого темного кольца определяет средний размер эритроцита на мазке крови, а отношение яркости первого темного и первого светлого колец связано с разбросом клеток по размерам [2]. Возникает вопрос, как влияет различие форм эритроцитов на дифракционную картину. Анализу этого вопроса посвящена настоящая работа. Основное внимание мы уделим расчету видности дифракционной картины, которая возникает при рассеянии лазерного пучка на ансамбле частиц, отличающихся друг от друга как по размерам, так и по формам.

Будем моделировать эритроцит на мазке крови плоским эллиптическим диском. Рассеяние лазерного пучка на таком диске описывается формулой [2]

$$
I=I_{0}|\gamma|^{2}\left(a b \frac{k}{z}\right)^{2}\left[\frac{J_{1}(q)}{q}\right]^{2} .
$$

Здесь $I_{0}-$ интенсивность падающего пучка, $|\gamma|^{2}-$ параметр, определяемый толщиной диска и его оптической плотностью, $a$ и $b-$ размеры полуосей диска, $k-$ волновое число световой волны, $z$ - расстояние от мазка крови до экрана наблюдения, $J_{1}(q)$ - функция Бесселя первого порядка. Аргумент этой функции определятся формулой

$$
q=\frac{k}{z} \sqrt{a^{2} x^{2}+b^{2} y^{2}},
$$

где $x, y$ - декартовы координаты точки на экране наблюдения. Формулы (2), (3) описывают распределение интенсивности света в той части экрана наблюдения, которая лежит за пределами прямого лазерного пучка. Оси координат на экране наблюдения направлены параллельно осям симметрии диска.

Интенсивность света в центре дифракционной картины найдем по формулам (2), (3), полагая $x \rightarrow 0, y \rightarrow 0$. Учитывая асимптотику функции Бесселя [27], получим

$$
I(0)=\frac{1}{4} I_{0}|\mathcal{\gamma}|^{2}\left(a b \frac{k}{z}\right)^{2} .
$$


Величину $I(0)$ удобно использовать в качестве масштаба интенсивности света в ДК. Интенсивность света в произвольной точке ДК, нормированная на интенсивность центрального дифракционного максимума, определяется формулой

$$
\frac{I}{I(0)}=\left[\frac{2 J_{1}(q)}{q}\right]^{2} .
$$

Здесь величина $q$ определяется формулой (3).

В области первого минимума интенсивности света на ДК функцию Бесселя можно аппроксимировать линейной функцией [27]

$$
J_{1}(q)=\beta\left(q-q_{1}\right) .
$$

Здесь

$$
\beta=-0.4, \quad q_{1}=3.82 .
$$

В этой области распределение нормированной интенсивности света приобретает вид

$$
\frac{1}{4 \beta^{2}} \frac{I}{I(0)}=\left(1-\frac{q_{1}}{q}\right)^{2} .
$$

Допустим, что рассматриваемый эллиптический диск характеризуется малым эксцентриситетом (т.е. мало отличается от круглого диска). В этом случае размеры полуосей диска можно представить в виде

$$
a=a_{0}\left(1+\varepsilon_{2}\right), \quad b=a_{0}\left(1-\varepsilon_{2}\right) .
$$

Здесь $\varepsilon_{2}$ - малый безразмерный параметр, такой что

$$
\left|\varepsilon_{2}\right| \ll 1 \text {. }
$$

Подставив (7) в (3) и учитывая (8), получим приближенное выражение для величины $q$, а именно

$$
q=\frac{k}{z} a_{0} \sqrt{x^{2}+y^{2}}\left(1+\varepsilon_{2} \frac{x^{2}-y^{2}}{x^{2}+y^{2}}\right) .
$$

Формулы (5), (6), (9) описывают рассеяние лазерного пучка на эллиптическом диске с малым эксцентриситетом.

Теперь предположим, что оси симметрии диска повернуты на некоторый угол $\varphi$ относительно декартовых осей координат на экране наблюдения. Тогда координаты $x, y$ в формуле (9) следует заменить на новые координаты в соответствии с правилом

$$
x \rightarrow x \cos \varphi+y \sin \varphi, \quad y \rightarrow-x \sin \varphi+y \cos \varphi .
$$

При этом выражение для величины $q$ приобретает вид

$$
q=\frac{k}{z} a_{0} \sqrt{x^{2}+y^{2}}\left[1+\varepsilon_{2} \frac{\left(x^{2}-y^{2}\right) \cos 2 \varphi+2 x y \sin 2 \varphi}{x^{2}+y^{2}}\right]
$$

или

$$
q=\sqrt{g}\left[1+\varepsilon_{2} \frac{h \cos 2 \varphi+2 u v \sin 2 \varphi}{g}\right]
$$

где введены величины

$$
u=\frac{k a_{0}}{z} x, \quad v=\frac{k a_{0}}{z} y
$$

и

$$
g=u^{2}+v^{2}, \quad h=u^{2}-v^{2} .
$$

Из формул (8), (11) следует, что величину $\frac{q_{1}}{q}$ можно приближенно записать в виде

$$
\frac{q_{1}}{q}=\frac{q_{1}}{\sqrt{g}}-\varepsilon_{2} \frac{q_{1}}{\sqrt{g}} \frac{h \cos 2 \varphi+2 u v \sin 2 \varphi}{g} .
$$

Подставив (14) в (6), получим

$$
\begin{aligned}
& \frac{1}{4 \beta^{2}} \frac{I}{I(0)}=\left(1-\frac{q_{1}}{\sqrt{g}}\right)^{2}+2 \varepsilon_{2} \frac{q_{1}}{\sqrt{g}}\left(1-\frac{q_{1}}{\sqrt{g}}\right) \\
& \times \frac{h \cos 2 \varphi+2 u v \sin 2 \varphi}{g}+\left[\varepsilon_{2} \frac{q_{1}}{\sqrt{g}} \frac{h \cos 2 \varphi+2 u v \sin 2 \varphi}{g}\right]^{2} .
\end{aligned}
$$

Формула (15) описывает рассеяние лазерного пучка на произвольно ориентированном эллиптическом диске с малым эксцентриситетом.

Теперь предположим, что угол поворота эллиптического диска относительно осей координат есть случайная величина с распределением плотности вероятности $w(\varphi)=\frac{1}{2 \pi}$. Это означает, что все ориентации диска равновероятны. Усредняя выражение (15) по углу $\varphi$, получим

$$
\frac{1}{4 \beta^{2}} \frac{\langle I\rangle_{\varphi}}{I(0)}=\left(1-\frac{q_{1}}{\sqrt{g}}\right)^{2}+\frac{1}{2} \varepsilon_{2}^{2}\left(\frac{q_{1}}{\sqrt{g}}\right)^{2} .
$$

При выводе (16) мы учли формулы (13), а также то, что

$$
\begin{gathered}
\langle\cos 2 \varphi\rangle_{\varphi}=\langle\sin 2 \varphi\rangle_{\varphi}=\langle\sin 4 \varphi\rangle_{\varphi}=0, \\
\left\langle\cos ^{2} 2 \varphi\right\rangle_{\varphi}=\left\langle\sin ^{2} 2 \varphi\right\rangle_{\varphi}=\frac{1}{2} .
\end{gathered}
$$

Итак, формула (16) описывает рассеяние лазерного пучка на хаотически ориентированном эллиптическом диске с малым эксцентриситетом.

Предположим далее, что параметр $\varepsilon_{2}$, характеризующий эксцентриситет диска, есть случайная величина с характеристиками

$$
\left|\varepsilon_{2}\right| \ll 1, \quad\left\langle\varepsilon_{2}\right\rangle=0, \quad\left\langle\varepsilon_{2}^{2}\right\rangle=\sigma_{2}^{2} .
$$

Тогда, усредняя выражение (16) по параметру $\varepsilon_{2}$, получим

$$
\frac{1}{4 \beta^{2}} \frac{\langle I\rangle_{\varphi, \varepsilon}}{I(0)}=\left(1-\frac{q_{1}}{\sqrt{g}}\right)^{2}+\frac{1}{2} \sigma_{2}^{2}\left(\frac{q_{1}}{\sqrt{g}}\right)^{2} .
$$

Формула (18) описывает рассеяние лазерного пучка на хаотически ориентированных эллиптических дисках со 
случайным эксцентриситетом. В этой формуле параметр $g$ определяется выражениями (12), (13). Используя эти формулы, получим

$$
\sqrt{g}=\frac{k a_{0}}{z} \sqrt{x^{2}+y^{2}} .
$$

Здесь величина $a_{0}$ характеризует размер эллиптического диска. Допустим теперь, что параметр $a_{0}$, характеризующий размер эллиптического диска, есть также случайная величина. Определим эту величину формулой

$$
a_{0}=R_{0}\left(1+\varepsilon_{1}\right) .
$$

Здесь величина $R_{0}$ характеризует средний размер эллиптических дисков, а параметр $\varepsilon_{1}$ есть случайная величина с характеристиками

$$
\left|\varepsilon_{1}\right| \ll 1, \quad\left\langle\varepsilon_{1}\right\rangle=0, \quad\left\langle\varepsilon_{1}^{2}\right\rangle=\sigma_{1}^{2} .
$$

Формулы (21) означают, что неоднородность ансамбля эллиптических дисков, моделирующих эритроциты на мазке крови, относительно мала. Дисперсия (разброс) размеров эритроцитов характеризуется величиной $\sigma_{1}^{2}$.

Подставляя (20) в (19), получим

$$
\sqrt{g}=\left(1+\varepsilon_{1}\right) \frac{k R_{0}}{z} \sqrt{x^{2}+y^{2}} .
$$

Отсюда

$$
\frac{q_{1}}{\sqrt{g}}=\frac{q_{1}}{\left(1+\varepsilon_{1}\right) \frac{k R_{0}}{z} \sqrt{x^{2}+y^{2}}}
$$

или

$$
\frac{q_{1}}{\sqrt{g}}=\frac{P_{0}}{1+\varepsilon_{1}}
$$

где

$$
P_{0}=\frac{q_{1} z}{k R_{0} \sqrt{x^{2}+y^{2}}} .
$$

Подставив (22) в (18), получим

$$
\frac{1}{4 \beta^{2}} \frac{\langle I\rangle_{\varphi, \varepsilon}}{I(0)}=\left(1-\frac{P_{0}}{1+\varepsilon_{1}}\right)^{2}+\frac{1}{2} \sigma_{2}^{2}\left(\frac{P_{0}}{1+\varepsilon_{1}}\right)^{2} .
$$

Учитывая (21), представим приближенное выражение в виде

$$
\frac{1}{4 \beta^{2}} \frac{\langle I\rangle_{\varphi, \varepsilon}}{I(0)}=\left(1-P_{0}+P_{0} \varepsilon_{1}\right)^{2}+\frac{1}{2} \sigma_{2}^{2} P_{0}^{2}\left(1-2 \varepsilon_{1}+\varepsilon_{1}^{2}\right)
$$

или

$$
\begin{aligned}
\frac{1}{4 \beta^{2}} \frac{\langle I\rangle_{\varphi, \varepsilon}}{I(0)}= & \left(1-P_{0}\right)^{2}+2 \varepsilon_{1} P_{0}\left(1-P_{0}\right)+\left(P_{0} \varepsilon_{1}\right)^{2} \\
& +\frac{1}{2} \sigma_{2}^{2} P_{0}^{2}\left(1-2 \varepsilon_{1}+\varepsilon_{1}^{2}\right) .
\end{aligned}
$$

Усредним это выражение по параметру $\varepsilon_{1}$. Получим

$$
\frac{1}{4 \beta^{2}} \frac{\langle I\rangle_{\varphi, \varepsilon, \varepsilon_{1}}}{I(0)}=\left(1-P_{0}\right)^{2}+\sigma_{1}^{2} P_{0}^{2}+\frac{1}{2} \sigma_{2}^{2} P_{0}^{2}\left(1+\mu_{1}\right)
$$

или приближенно

$$
\frac{1}{4 \beta^{2}} \frac{\langle I\rangle_{\varphi, \varepsilon, \varepsilon_{1}}}{I(0)}=\left(1-P_{0}\right)^{2}+\left(\sigma_{1}^{2}+\frac{1}{2} \sigma_{2}^{2}\right) P_{0}^{2} .
$$

Формула (23) описывает распределение нормированной интенсивности света вблизи первого темного кольца ДК, возникающей при рассеянии лазерного пучка на мазке крови. Она учитывает разброс эритроцитов по размерам, формам и ориентациям в пространстве. В этой формуле $\langle I\rangle_{\varphi, \varepsilon, \varepsilon_{1}}$ - интенсивность света в данной точке экрана наблюдения, $\beta=-0.4, q_{1}=3.82-$ постоянные величины (параметры функции Бесселя), $I(0)$ - интенсивность света в центре ДК, $\sigma_{2}^{2}$ - мера разброса эритроцитов по формам, $\sigma_{1}^{2}-$ мера разброса эритроцитов по размерам. Параметр $P_{0}$ определяется формулой

$$
P_{0}=\frac{q_{1} z}{k R_{0} \sqrt{x^{2}+y^{2}}}
$$

где $x, y$ - координаты точки на экране наблюдения, $R_{0}$ - характеристика среднего размера эритроцитов, $k-$ волновое число световой волны, $z-$ расстояние от мазка крови до экрана наблюдения.

Как видно из формулы (23), нормированная интенсивность света в первом минимуме ДК достигается при условии

$$
P_{0}=1
$$

и определяется формулой

$$
\frac{1}{4 \beta^{2}} \frac{\langle I\rangle_{\min }}{I(0)}=\sigma_{1}^{2}+\frac{1}{2} \sigma_{2}^{2} .
$$

Одним из параметров ДК, который может быть измерен экспериментально, является видность этой картины в районе первого минимума интенсивности света (первого темного кольца). Определим этот параметр формулой (1). Если учитывать неоднородность ансамбля только по размерам частиц, то видность ДК в районе первого минимума интенсивности света приближенно выражается формулой [2]

$$
v=1-76 \sigma_{1}^{2} .
$$

Эта формула верна в пределе слабой неоднородности ансамбля, когда

$$
\sigma_{1}^{2}=\left\langle\varepsilon_{1}^{2}\right\rangle=\frac{\left\langle(R-\langle R\rangle)^{2}\right\rangle}{\langle R\rangle^{2}} \equiv \frac{\sigma_{R}^{2}}{\langle R\rangle^{2}} \ll 1 .
$$

Если же учитывать неоднородность ансамбля эритроцитов как по размерам, так и по формам клеток, то формула для видности ДК приобретает вид

$$
v=1-76 \sigma^{2} .
$$

Здесь

$$
\sigma^{2}=\sigma_{1}^{2}+\frac{1}{2} \sigma_{2}^{2}
$$


$\sigma_{1}^{2}$ - мера разброса эритроцитов по размерам, $\sigma_{2}^{2}-$ мера разброса эритроцитов по формам, $\sigma^{2}-$ комбинированный параметр, характеризующий разброс эритроцитов как по размерам, так и по формам. Из формулы (28) следует, что

$$
\sigma=\sqrt{\frac{1-v}{76}}
$$

Эта формула представляет собой основной результат расчета. Она связывает видность дифракционной картины $v$ в районе первого минимума интенсивности света с параметром неоднородности ансамбля эритроцитов по размерам и формам $\sigma$ для исследуемого образца крови. Формула (30) применима для слабо неоднородных ансамблей эритроцитов. Таким образом, измеряя видность дифракционной картины, возникающей при рассеянии лазерного пучка на мазке крови, и используя формулу (30), можно оценить разброс эритроцитов по размерам и формам в исследуемом образце крови.

\section{Мера асимметрии эритроцита}

В этой части работы мы введем меру асимметрии эритроцита на мазке крови без предположения о какойлибо конкретной форме эритроцита. Это позволит количественно оценивать асимметрию реальных эритроцитов на мазке крови. Такую меру можно ввести на основании сравнения формы эритроцита с равновеликим (по площади) кругом. Определим эту меру так

$$
M=\frac{\Delta S}{S}=\frac{S-S_{\text {об }}}{S},
$$

где $S_{\text {об }}$ - площадь общей части эритроцита на мазке крови и равновеликого ему круга, $S$ - площадь круга, равная площади эритроцита. При этом предполагаем, что круг расположен относительно фигуры, изображающей эритроцит, так, что площадь общей части фигуры и круга является максимально возможной. В частности, мера асимметрии круглого эритроцита равна нулю.

Применим это определение к фигуре, имеющей форму эллипса, и установим связь меры асимметрии $M$, определяемой формулой (31), с параметром асимметрии эллипса $\varepsilon_{2}$, определяемым формулами (7). Расчет показывает, что эта связь выражается формулой (см. Приложение)

$$
\varepsilon_{2}=\frac{\pi}{2} M
$$

Из формулы (32) следует, что

$$
\sigma_{2}^{2}=\left\langle\varepsilon_{2}^{2}\right\rangle=\frac{\pi^{2}}{4}\left\langle M^{2}\right\rangle .
$$

Здесь угловые скобки обозначают усреднение по ансамблю эритроцитов. Подставив (33) в (29), получим

$$
\sigma^{2}=\sigma_{1}^{2}+\frac{\pi^{2}}{8}\left\langle M^{2}\right\rangle
$$

Формулы (28) и (34) применимы для слабо неоднородных ансамблей эритроцитов. Параметры $v, \sigma_{1}^{2}$ и $M$ в этих формулах определены формулами (1), (27), (31). В явном виде параметры $\sigma_{1}^{2}$ и $\left\langle M^{2}\right\rangle$ можно записать так:

$$
\sigma_{1}^{2}=\frac{\left\langle(R-\langle R\rangle)^{2}\right\rangle}{\langle R\rangle^{2}}, \quad\left\langle M^{2}\right\rangle=\left\langle\left(1-\frac{S_{\text {об }}}{S}\right)^{2}\right\rangle,
$$

где

$$
\begin{aligned}
\langle R\rangle & =\frac{1}{N} \sum_{i=1}^{N} R_{i}, \\
\left\langle(R-\langle R\rangle)^{2}\right\rangle & =\frac{1}{N} \sum_{i=1}^{N}\left(R_{i}-\langle R\rangle\right)^{2}, \\
\left\langle\left(1-\frac{S_{\text {об }}}{S}\right)^{2}\right\rangle & =\frac{1}{N} \sum_{i=1}^{N}\left(1-\frac{S_{\text {об } i}}{S_{i}}\right)^{2}, \\
S_{i} & =\pi R_{i}^{2} .
\end{aligned}
$$

Здесь индекс , $i^{\text {“ }}$ нумерует отдельные эритроциты на мазке крови, $N$ - полное число эритроцитов на мазке, $S_{i}$ - площадь эритроцита на мазке, $R_{i}-$ радиус эритроцита, $\langle R\rangle-$ средний радиус эритроцита, $S_{\text {об } i}$ - максимальная площадь общей части эритроцита и равновеликого круга. Параметр $\sigma^{2}$, определяемый формулой (34), можно рассматривать как меру неоднородности образца крови по размерам и формам. Как видно из формулы (30), параметр $\sigma$ простым образом связан с видностью дифракционной картины $v$, возникающей при рассеянии лазерного пучка на мазке крови. Таким образом, проведенный анализ показывает, что неоднородность образца крови по размерам и формам эритроцитов может быть экспериментально измерена методом лазерной дифрактометрии.

\section{Численные расчеты}

В этом разделе мы проведем численное моделирование дифракционной картины, возникающей при рассеянии лазерного пучка на неоднородном ансамбле эритроцитов, и найдем зависимость видности этой картины от параметра неоднородности ансамбля эритроцитов $v=v(\sigma)$.

Будем считать параметры $\varepsilon_{1}$ и $\varepsilon_{2}$ нашей теоретической модели дискретными случайными величинами с идентичными характеристиками. А именно

$$
\begin{aligned}
& \varepsilon_{1 i}=\Delta \varepsilon i, \quad p_{i}=\frac{1}{N}, \quad-\frac{N}{2} \leq i \leq \frac{N}{2}, \\
& \varepsilon_{2 j}=\Delta \varepsilon j, \quad p_{j}=\frac{1}{N}, \quad-\frac{N}{2} \leq j \leq \frac{N}{2} .
\end{aligned}
$$

При этом средние значения и дисперсии этих величин определяются формулами

$$
\left\langle\varepsilon_{1}\right\rangle=\left\langle\varepsilon_{2}\right\rangle=\sum_{i} \varepsilon_{1 i} p_{i}=\frac{1}{N} \sum_{i=-N / 2}^{N / 2} \varepsilon_{1 i}=\frac{\Delta \varepsilon}{N} \sum_{i=-N / 2}^{N / 2} i=0,
$$




$$
\begin{aligned}
\sigma_{1}^{2} & =\sigma_{2}^{2}=\left\langle\varepsilon_{1}^{2}\right\rangle=\sum_{i} \varepsilon_{1 i}^{2} p_{i}=\frac{1}{N} \sum_{i=-N / 2}^{N / 2} \varepsilon_{1 i}^{2} \\
& =(\Delta \varepsilon)^{2} \frac{1}{N} \sum_{i=-N / 2}^{N / 2} i^{2} .
\end{aligned}
$$

Для численных расчетов положим $N=10$. Тогда

$$
\frac{1}{N} \sum_{i=-N / 2}^{N / 2} i^{2}=\frac{2}{N} \sum_{i=1}^{N / 2} i^{2}=\frac{1}{5} \sum_{i=1}^{5} i^{2}=11,
$$

$\sigma_{1}^{2}=11(\Delta \varepsilon)^{2} \quad$ и $\quad \Delta \varepsilon=\sigma_{1} / \sqrt{11}$. Теперь используем формулу $\sigma^{2}=\sigma_{1}^{2}+\frac{1}{2} \sigma_{2}^{2}$. Полагая $\sigma_{1}^{2}=\sigma_{2}^{2}$, получим $\sigma_{1}=\sigma \sqrt{2 / 3}$ и $\Delta \varepsilon=\sigma \sqrt{\frac{2}{33}}$. Тем самым значения параметров $\varepsilon_{1}$ и $\varepsilon_{2}$, а вместе с ними характеристики ансамбля эритроцитов полностью определены. Используя эти данные, решим прямую задачу дифракции и найдем видность дифракционной картины.

Введем функцию

$$
G(x)=\left[\frac{2 J_{1}(x)}{x}\right]^{2}
$$

Распределение интенсивности света в дифракционной картине найдем по формуле

$$
f(u, v)=\frac{I}{I(0)}=\langle G(q)\rangle=\frac{1}{N^{2} M} \sum_{i=-N / 2}^{N / 2} \sum_{j=-N / 2}^{N / 2} \sum_{k=1}^{M} G\left(q_{i j k}\right),
$$

где

$$
\begin{aligned}
& q_{i j k}=\left(1+\varepsilon_{1 j}\right) \times \\
& \sqrt{\left(1+\varepsilon_{i}\right)^{2}\left(u \cos \varphi_{k}+v \sin \varphi_{k}\right)^{2}+\left(1-\varepsilon_{i}\right)^{2}\left(-u \sin \varphi_{k}+v \cos \varphi_{k}\right)^{2}}
\end{aligned}
$$

и

$$
\varphi_{k}=\Delta \varphi k, \quad \Delta \varphi=\frac{2 \pi}{M}, \quad 1 \leq k \leq M
$$

При выводе этих формул мы использовали формулы (10). Нормированные координаты $u$ и $v$ определяются формулами

$$
u=\frac{k R_{0}}{z} x, \quad v=\frac{k R_{0}}{z} y .
$$

Угол $\varphi$ задает ориентацию эллипса, моделирующего эритроцит, на мазке крови. Мы считаем этот угол дискретной случайной величиной с равномерной функцией распределения. Таким образом, мы имитируем ситуацию, когда ориентации клеток на мазке крови полностью случайны. Заметим, что распределение интенсивности света в дифракционной картине обладает круговой симметрией. Поэтому его можно представить в виде распределения интенсивности света на отдельной секущей линии, т. е. на линии, идущей от центра картины в ее периферии. В частности, секущую линию можно направить вдоль

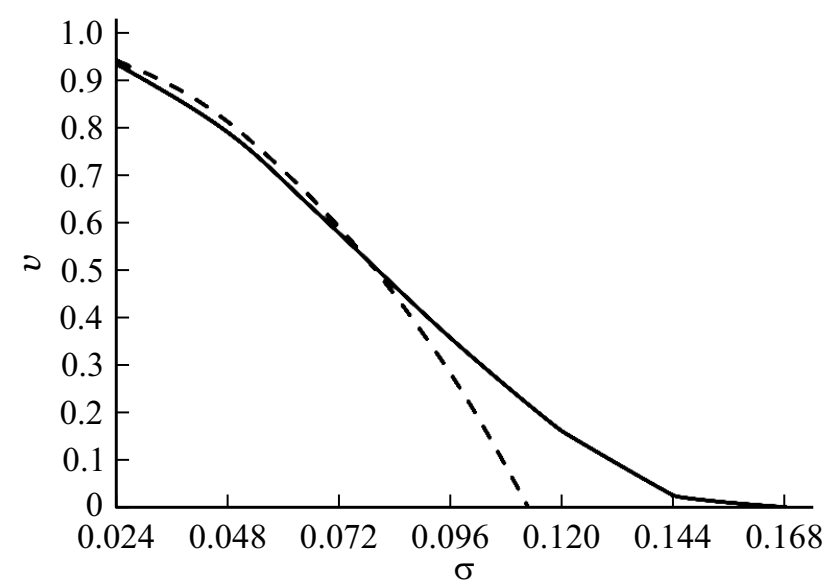

Рис. 2. Зависимость видности дифракционной картины от неоднородности ансамбля эритроцитов. Сплошная кривая построена численно (см. текст). Штриховая линия построена по формуле (28).

горизонтальной оси координат на экране наблюдения. Тогда мы получим функцию

$$
f(u)=\frac{1}{N^{2} M} \sum_{i=-N / 2}^{N / 2} \sum_{j=-N / 2}^{N / 2} \sum_{k=1}^{M} G\left(q_{i j k}\right),
$$

где

$$
\begin{aligned}
q_{i j k}= & \left(1+\varepsilon_{1 j}\right) \\
& \times \sqrt{\left(1+\varepsilon_{i}\right)^{2}\left(u \cos \varphi_{k}\right)^{2}+\left(1-\varepsilon_{i}\right)^{2}\left(-u \sin \varphi_{k}\right)^{2}} .
\end{aligned}
$$

В численных расчетах мы полагали $N=10$ и $M=100$. Это значит, что мы рассчитываем дифракционную картину от ансамбля в $N^{2} M=10^{4}$ эритроцитов для каждого значения параметра $\sigma$. Один такой расчет дает нам одно значение видности ДК и, следовательно, одну точку зависимости $v=v(\sigma)$. Отметим, что точность измерения видности зависит от разрешения дифракционной картины. В наших расчетах мы полагали разрешение равным $10^{6}$ пикселей. Такое разрешение обеспечивает достаточно высокую точность и на практике может быть достигнуто при использовании стандартной регистрирующей аппаратуры. Повторяя расчет для других значений параметра $\sigma$, мы построили всю зависимость $v=v(\sigma)$. Эта зависимость показана на рис. 2. Для сравнения на этом же рисунке штрихами показана линия, построенная по формуле (28). Видно, что для слабо неоднородных ансамблей эритроцитов эта формула дает хорошую аппроксимацию зависимости видности дифракционной картины от параметра неоднородности ансамбля эритроцитов.

Кроме того, мы по отдельности рассмотрели случаи, когда ансамбль неоднороден только по размерам или только по формам клеток. Результаты этих расчетов представлены на рис. 3. Из этого рисунка видно, что 

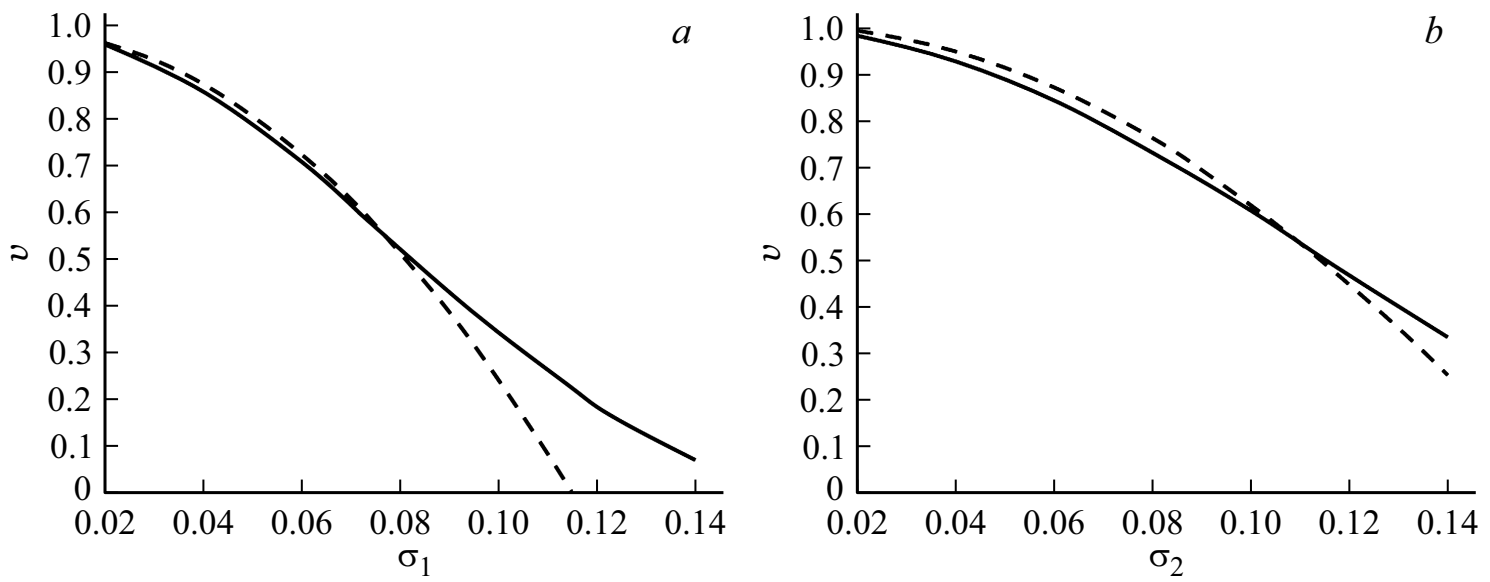

Рис. 3. Зависимость видности дифракционной картины от неоднородности ансамбля эритроцитов. Сплошная кривая на рис. $a$ построена с учетом разброса частиц только по размерам $\sigma_{1}$. Сплошная кривая на рис. $b$ построена с учетом разброса частиц только по формам $\sigma_{2}$. Штриховые линии построены по формуле $(28)$ при $\sigma_{2}=0(a)$ и при $\sigma_{1}=0$.

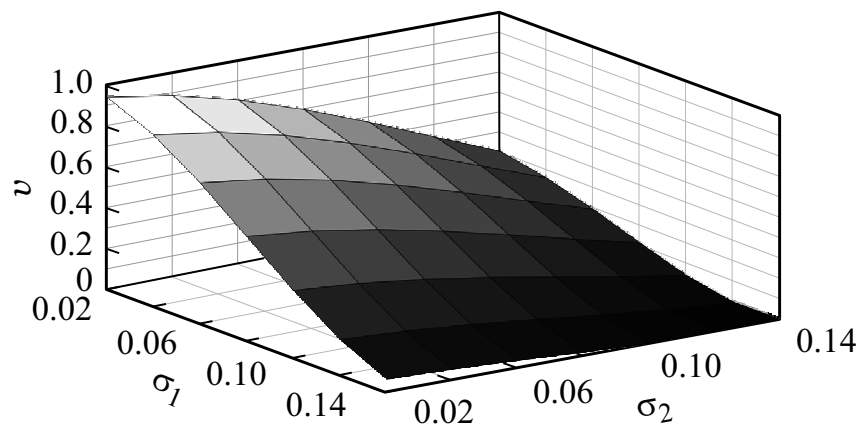

Рис. 4. Видность дифракционной картины как функция параметров, характеризующих неоднородность ансамбля эритроцитов по размерам $\left(\sigma_{1}\right)$ и по форме $\left(\sigma_{2}\right)$.

Видность дифракционной картины как функция параметров, характеризующих разброс эритроцитов по размерам $\left(\sigma_{1}\right)$ и формам $\left(\sigma_{2}\right)$

\begin{tabular}{c|c|c|c|c|c|c|c}
\hline \multirow{2}{*}{$\sigma_{2}$} & \multicolumn{7}{|c}{$\sigma_{1}$} \\
\cline { 2 - 8 } & 0.02 & 0.04 & 0.06 & 0.08 & 0.10 & 0.12 & 0.14 \\
\hline 0.14 & 0.3187 & 0.2715 & 0.2016 & 0.1208 & 0.0458 & 0.0000 & - \\
\hline 0.12 & 0.4492 & 0.3901 & 0.3021 & 0.1994 & 0.0995 & 0.0227 & - \\
\hline 0.10 & 0.5817 & 0.5110 & 0.4054 & 0.2814 & 0.1589 & 0.0581 & 0.0000 \\
\hline 0.08 & 0.7049 & 0.6238 & 0.5025 & 0.3597 & 0.2171 & 0.0967 & 0.0180 \\
\hline 0.06 & 0.8118 & 0.7220 & 0.5873 & 0.4281 & 0.2684 & 0.1319 & 0.0379 \\
\hline 0.04 & 0.8942 & 0.7977 & 0.6529 & 0.4814 & 0.3087 & 0.1601 & 0.0551 \\
\hline 0.02 & 0.9464 & 0.8456 & 0.6942 & 0.5150 & 0.3344 & 0.1783 & 0.0666
\end{tabular}

оценка (28) видности дифракционной картины, показанная штриховой линией, хорошо совпадает с результатами численного расчета в области достаточно малых значений $\sigma_{1}$ и $\sigma_{2}$, т. е. для слабо неоднородных ансамблей эритроцитов.

Зависимость видности дифракционной картины от неоднородности ансамбля эритроцитов как по размерам, так и по формам, показана на рис. 4. На этом рисунке видность изображена в виде поверхности в трехмерном пространстве, а по декартовым осям координат в горизонтальной плоскости отложены значения параметров $\sigma_{1}$ и $\sigma_{2}$.

Приводим также числовую таблицу, соответствующую рис. 4. Прочерки сигнализируют о том, что темное кольцо в силу большого значения разброса эритроцитов по размерам и формам настолько размыто, что локального минимума интенсивности в районе темного кольца больше нет, а значит понятие видности дифракционной картины теряет смысл.

\section{Заключение}

В настоящей работе построена аналитическая модель рассеяния лазерного пучка на неоднородном ансамбле эритроцитов. Модель учитывает разброс эритроцитов по размерам, формам и ориентациям в пространстве. Установлена связь экспериментально измеряемого параметра - видности дифракционной картины - с характеристикой, имеющей смысл меры неоднородности образца крови по размерам и формам эритроцитов. При условии независимого измерения разброса эритроцитов по размерам (например, с помощью счетчика Коултера), с помощью лазерной дифрактометрии можно оценить неоднородность исследуемого мазка крови по формам клеток. Такие измерения могут быть полезны при диагностике заболеваний, связанных с нарушениями форм клеток крови.

В данной работе под распределением по размерам мы понимаем распределение эритроцитов по диаметру. 
В модели плоских дисков, которую мы используем, распределение эритроцитов по толщине не влияет существенным образом на вид дифракционной картины, а влияет лишь на ее яркость. На вид дифракционной картины влияет рельеф поверхности эритроцита. Однако анализ этого вопроса требует дополнительного исследования.

\section{Благодарности}

Авторы благодарны А.В. Приезжеву и А.Е. Луговцову за обсуждение результатов работы.

\section{Финансирование работы}

Работа поддержана грантом Российского фонда фундаментальных исследований № 17-29-03507.

\section{Конфликт интересов}

Авторы заявляют, что у них нет конфликта интересов.

\section{Приложение}

Рассмотрим эллипс с полуосями $a$ и $b$, и равновеликий круг радиуса $\sqrt{a b}$. Центр эллипса совпадает с центром круга. Точку пересечения круга и эллипса в области $x>0, y>0$ будем характеризовать декартовыми координатами $x, y$, а также полярными координатами $r, \varphi$. Тогда

$$
\begin{gathered}
\frac{x^{2}}{a^{2}}+\frac{y^{2}}{b^{2}}=1, \\
x^{2}+y^{2}=a b, \\
\operatorname{tg} \varphi=\frac{y}{x}, \\
x=r \cos \varphi, \quad y=r \sin \varphi .
\end{gathered}
$$

Введем также вспомогательный угол $\psi$, определив его формулами

$$
\sin \psi=\frac{y}{b}, \quad \cos \varphi=\frac{x}{a} .
$$

Полярный угол $\varphi$ и вспомогательный угол $\psi$ связаны соотношением

$$
\frac{y}{x}=\operatorname{tg} \varphi=\frac{b}{a} \operatorname{tg} \psi
$$

Уравнение эллипса в полярных координатах

$$
r(\varphi)=\frac{a b}{\sqrt{a^{2} \sin ^{2} \varphi+b^{2} \cos ^{2} \varphi}} .
$$

Вычислим площадь сектора эллипса. Используя полярные координаты, получим

$$
\begin{aligned}
S_{\text {эл }} & =\int_{0}^{\varphi} d \varphi \int_{0}^{r(\varphi)} r d r=\frac{1}{2} \int_{0}^{\varphi} r^{2}(\varphi) d \varphi \\
& =\frac{1}{2} a^{2} b^{2} \int_{0}^{\varphi} \frac{d \varphi}{a^{2} \sin ^{2} \varphi+b^{2} \cos ^{2} \varphi} .
\end{aligned}
$$

Для вычисления этого интеграла перейдем к переменной $\psi$.

Используем формулы

$$
\begin{gathered}
\sin ^{2} \varphi=\frac{\operatorname{tg}^{2} \varphi}{1+\operatorname{tg}^{2} \varphi}=\frac{\frac{b^{2}}{a^{2}} \operatorname{tg}^{2} \psi}{1+\frac{b^{2}}{a^{2}} \operatorname{tg}^{2} \psi}=\frac{b^{2} \sin ^{2} \psi}{a^{2} \cos ^{2} \psi+b^{2} \sin ^{2} \psi}, \\
\cos ^{2} \varphi=\frac{1}{1+\operatorname{tg}^{2} \varphi}=\frac{1}{1+\frac{b^{2}}{a^{2}} \operatorname{tg}^{2} \psi}=\frac{a^{2} \cos ^{2} \psi}{a^{2} \cos ^{2} \psi+b^{2} \sin ^{2} \psi}, \\
a^{2} \sin ^{2} \varphi+b^{2} \cos ^{2} \varphi=\frac{a^{2} b^{2}}{a^{2} \cos ^{2} \psi+b^{2} \sin ^{2} \psi}, \\
d \operatorname{tg} \varphi=\frac{d \varphi}{\cos ^{2} \varphi}=\frac{b}{a} d \operatorname{tg} \psi=\frac{b}{a} \frac{d \psi}{\cos ^{2} \psi}, \\
\frac{d \varphi}{a} \frac{\cos ^{2} \varphi}{\cos ^{2} \psi} d \psi=\frac{b}{a} \frac{a^{2} \cos ^{2} \psi}{a^{2} \cos ^{2} \psi+b^{2} \sin ^{2} \psi} \frac{d \psi}{\cos ^{2} \psi+b^{2} \sin ^{2} \psi} d \psi \\
\frac{d \varphi}{a^{2} \sin ^{2} \varphi+b^{2} \cos ^{2} \varphi}=\frac{a^{2} \cos ^{2} \psi+b^{2} \sin ^{2} \psi}{a^{2} b^{2}} \\
\times \frac{a b}{a^{2} \cos ^{2} \psi+b^{2} \sin ^{2} \psi} d \psi=\frac{d \psi}{a b}
\end{gathered}
$$

Отсюда

$$
S_{e l}=\frac{1}{2} a^{2} b^{2} \int_{0}^{\varphi} \frac{d \varphi}{a^{2} \sin ^{2} \varphi+b^{2} \cos ^{2} \varphi}=\frac{1}{2} a b \int_{0}^{\psi} d \psi=\frac{1}{2} a b \psi .
$$

Итак, площадь сектора эллипса с полуосями $a$ и $b$ выражается формулой

$$
S_{e l}=\frac{1}{2} a b \psi
$$

Здесь угол $\psi$ связан с полярным углом $\varphi$ формулой (П1). Площадь сектора круга радиуса $\sqrt{a b}$, соответствующего полярному углу $\varphi$, есть

$$
S_{\text {circ }}=\frac{1}{2} a b \varphi .
$$

Теперь найдем координаты точки пересечения эллипса с равновеликим кругом. Для этого возьмем уравнения эллипса и окружности

$$
\frac{x^{2}}{a^{2}}+\frac{y^{2}}{b^{2}}=1, \quad x^{2}+y^{2}=a b
$$

и решим их относительно величин $x$ и $y$. Получим

$$
x=a \sqrt{\frac{b}{a+b}}, \quad y=b \sqrt{\frac{a}{a+b}} .
$$

Эти формулы определяют декартовы координаты точек пересечения эллипса с равновеликим кругом. Угловая координата этой точки $\varphi$ определяется формулой

$$
\operatorname{tg} \varphi=\frac{y}{x} .
$$




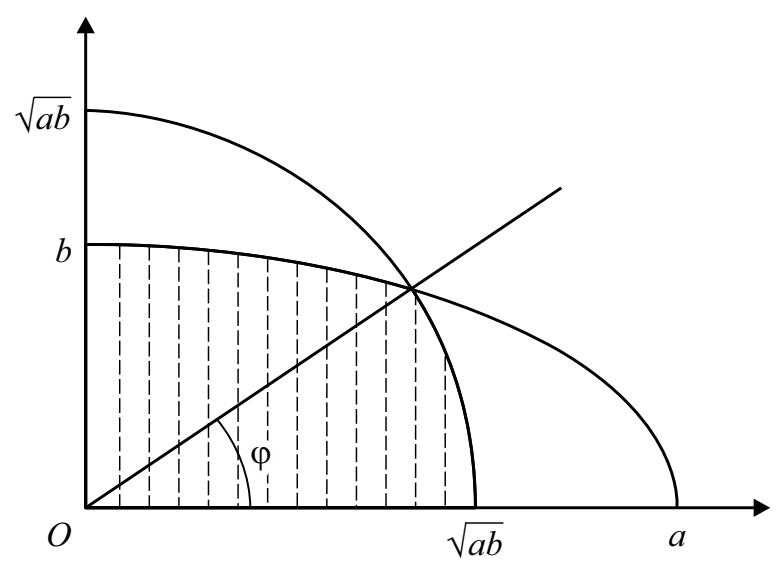

Рис. 5. Дуги окружности и равновеликого по площади эллипса. Заштрихована общая часть эллипса и круга.

Отсюда

$$
\operatorname{tg} \varphi=\frac{y}{x}=\frac{b \sqrt{a}}{a \sqrt{b}}=\sqrt{\frac{b}{a}} .
$$

При этом

$$
\operatorname{tg} \psi=\frac{a}{b} \operatorname{tg} \varphi=\sqrt{\frac{a}{b}} .
$$

Вычислим параметр асимметрии эллипса $M$, определяемый формулой (31). В силу симметрии достаточно рассмотреть часть картины, лежащую в области $x>0$ и $y>0$. Будем считать, для определенности, что $a>b$. Контуры эллипса и окружности для этого случая показаны на рис. 5.

На этом рисунке заштрихована общая часть эллипса и окружности. Незаштрихованные части эллипса и круга имеют одинаковую площадь $\Delta S$, которая является мерой асимметрии эллипса. Из рисунка видно, что эту площадь можно найти как разность площадей секторов эллипса и круга, соответствующих одному и тому же полярному углу $\varphi$, т. е.

$$
\Delta S=S_{\text {эл }}-S_{\text {кр }} .
$$

Используя формулы (П2) и (П3), находим

$$
\Delta S=\frac{1}{2} a b(\psi-\varphi) .
$$

Площадь четверти окружности $S=\frac{1}{4} \pi a b$. Отсюда

$$
M=\frac{\Delta S}{S}=\frac{2}{\pi}(\psi-\varphi) .
$$

Итак, получили

$$
M=\frac{2}{\pi}(\psi-\varphi)
$$

где

$$
\operatorname{tg} \varphi=\sqrt{\frac{b}{a}}, \quad \operatorname{tg} \psi=\sqrt{\frac{a}{b}} .
$$

Это верно для эллипса с произвольным эксцентриситетом.
Теперь рассмотрим частный случай эллипса с малым эксцентриситетом. Найдем связь параметра $M$, который может быть измерен для реального эритроцита, с параметром $\varepsilon_{2}$, фигурирующим в нашей теоретической модели и определяемым формулами (7). Считая, что $\left|\varepsilon_{2}\right| \ll 1$, получим

$$
\operatorname{tg} \varphi=\sqrt{\frac{b}{a}}=\sqrt{\frac{1-\varepsilon_{2}}{1+\varepsilon_{2}}} \approx \sqrt{\left(1-\varepsilon_{2}\right)^{2}}=1-\varepsilon_{2}
$$

и

$$
\operatorname{tg} \psi=\sqrt{\frac{a}{b}}=\sqrt{\frac{1+\varepsilon_{2}}{1-\varepsilon_{2}}} \approx \sqrt{\left(1+\varepsilon_{2}\right)^{2}}=1+\varepsilon_{2} .
$$

Следовательно, углы $\varphi$ и $\psi$ близки к $45^{\circ}$. Представим эти углы в виде

$$
\varphi=\frac{\pi}{4}-\alpha, \quad \psi=\frac{\pi}{4}+\beta,
$$

где $\alpha, \beta \ll 1$. Тогда

$$
\operatorname{tg} \varphi=1-2 \alpha, \quad \operatorname{tg} \psi=1+2 \beta .
$$

Из (П7), (П8), (П10) следует, что

$$
\alpha=\frac{\varepsilon_{2}}{2}, \quad \beta=\frac{\varepsilon_{2}}{2} .
$$

Используя (П9) и (П11), находим

$$
\psi-\varphi=\alpha+\beta=\varepsilon_{2} .
$$

Подставив это выражение в формулу (П5), получим $M=\frac{2}{\pi} \varepsilon_{2}$, откуда

$$
\varepsilon_{2}=\frac{\pi}{2} M
$$

\section{Список литературы}

[1] Юрчук Ю.С., Устинов В.Д., Никитин С.Ю., Приезжев А.В. // Квант. электрон. 2016. Т. 46. № 6. C. 515; Yurchuk Yu.S., Ustinov V.D., Nikitin S.Yu., Priezzhev A.V. // Quantum Electron. 2016. V. 46. N 6 P. 515. doi 10.1070/QEL16108

[2] Никитин С.Ю., Луговцов А.Е., Приезжеев А.В., Устинов В.Д. // Квант. электрон. 2011. Т. 41. № 9. C. 843; Nikitin S.Yu., Lugovtsov A.E., Priezzhev A.V., Ustinov V.D. // Quantum Electron. 2011. V. 41. N 9. P. 843. doi 10.1070/QE2011v041n09ABEH014675

[3] Patel K.V., Ferrucci L., Ershler W.B., Longo D.L., Guralnik J.M. // Archives of internal medicine. 2009. V. 169. N 5. P. 515.

[4] Titcomb C.P. // On the Risk. 2017. V. 33. N 1. P. 30.

[5] Foy B.H., Carlson J.C.T., Reinertsen E., Valls R.P.I, Pallares Lopez R., Palanques-Tost E., Mow C., Westover M.B., Aguirre A.D., Higgins J.M // JAMA Netw Open. 2020. V. 3. N 9 e2022058. doi 10.1001/jamanetworkopen.2020.22058

[6] Шифрин К.С. Введение в оптику океана. Л.: Гидрометеоиздат, 1983. $280 \mathrm{c.}$ 
[7] $X u \quad R$. Particle characterization: light scattering methods. Springer, 2002. doi 10.1007/0-306-47124-8

[8] Козинеи, Г.И., Погорелов В.М., Шмаров Д.А., Боев С.Ф., Сазонов В.В. Клетки крови - современные технологии их анализа. М.: Триада-Фарм, 2002. 200 с.

[9] Лопатин В.Н., Приезжев А.В., Апонасенко И.В., Шепелевич А.Д., Лопатин Н.В., Пожиленкова В.В., Простакова П.В. Методы светорассеяния в анализе дисперсных биологических сред. М.: Физматлит, 2004. 384 с.

[10] Yang Y., Zhang Z., Yang X., Yeo J.H., Jiang L., Jiang D. // J. Biomed. Opt. 2004. V. 9. N 5. P. 995-1001. doi $10.1117 / 1.1782572$

[11] Устинов В.Д. // Матем. моделирование. 2017. Т. 9. № 5. C. 561-569; Ustinov V.D. // Mathem. Models and Comp. Simulations. 2017. V. 9. N 5. P. 561-569. doi 10.1134/S2070048217050131

[12] Nikitin S.Yu., Priezzhev A.V., Lugovtsov A.E., Ustinov V.D., Razgulin A.V. // J. Quant. Spectrosc. Radiat. Transf. 2014. V. 146. P. 365. doi 10.1016/j.jqsrt.2014.05.012

[13] Ustinov V.D., Tsybrov E.G. // Inverse Problems in Science and Engineering. 2020. V. 28. N 11. P. 1633. doi 10.1080/17415977.2020.1761801

[14] Yurkin M.A., Hoekstra A.G. // J. Quant. Spectrosc. Radiat. Transf. 2011. V. 112. N 13. P. 2234. doi 10.1016/j.jqsrt.2011.01.031

[15] Yurkin M.A., Hoekstra A.G., Brock R.S., Lu J.Q. // Opt. Express. 2007. V. 15. N 26. P. 17902. doi 10.1364/OE.15.017902

[16] Eremin Y., Eremina E., Wriedt T. // Opt. Commun. 2005. V. 244. P. 15. doi 10.1016/j.optcom.2004.09.037

[17] Wriedt T., Hellmers J., Eremina E., Schuh R. // J. Quant. Spectrosc. Radiat. Transf. 2006. V. 100. N 1. P. 444. doi 10.1016/j.jqsrt.2005.11.057

[18] Электронный ресурс. Режим доступа: https://www.malvernpanalytical.com/ru/products/productrange/mastersizer-range

[19] Тихонов А.Н., Гласко В.Б. // Журн. вычисл. матем. и матем. физ. 1964. Т. 4. № 3. С. 564; Tikhonov A.N., Glasko V.B. // USSR Comput. Math. Math. Phys.1964. V. 4. N 3. P. 236. doi 10.1016/0041-5553(64)90254-X

[20] Tikhonov A.N., Goncharsky A.V., Stepanov V.V., Yagola A.G. Numerical methods for the solution of ill-posed problems. Springer, 2013. 328 p. doi 10.1007/978-94-015-8480-7

[21] Riley J.B., Agrawal Y.C. // Appl. Opt. 1991. V. 30. N 33. P. 4800. doi 10.1364/AO.30.004800

[22] Никитин С.Ю., Луговцов А.Е., Приезжеев А.В. // Квант. электрон. 2010. T. 40. № 12. C. 1074; Nikitin S.Yu., Lugovtsov A.E., Priezzhev A.V. // Quantum Electron. 2010. V. 40. N 12. P. 1074. doi 10.1070/QE2010v040n12ABEH014504

[23] Ахманов С.А., Никитин С.Ю. Физическая оптика. М.: Наука, 2004. 654 c.; Akhmanov S.A., Nikitin S.Yu. Physical optics. Oxford: Oxford University Press, 1997. 654 p.

[24] Саркисян Г.П., Саркисян А.Г., Даниелян А.М. // Изв. НАН Армении. Физика. 2019. Т. 54. № 2. С. 293.

[25] Саркисян Г.П. // Биолог. журн. Армении. 2020. Т. 72. № 12. C. 102.

[26] Саркисян Г.П. // Доклады НАН Армении. 2020. Т. 120. № 1. C. 45.

[27] Янке Е., Эмде Ф., Леш Ф. Специальные функции. М.: Наука, 1977; Janke E., Emde F., Lösch F. Tafeln Höherer Funktionen (Tables of Higher Functions). Stuttgart: Teubner, 1960; New York: MacGraw Hill, 1960; M.: Nauka, 1977. 